Heim, Ernst Ludwig: Georg Wilhelm Kessler, Der alte Heim. 2. Auflage, Leipzig 1846. IM'нотеP: GoRCE et MoRTIER, Histoire des religions. 1948.

Kussmaul, Adolf, Jugenderinnerungen eines alten Arztes. 6. Auflage, Stuttgart 1903.

Lebert, Hermann, Biographische Notizen. Breslau 1869.

Lettsom, John C.: R. Hingston Fox, Dr. Fothergill and his Friends. London 1919.

- James Johnston Abraham, Lettsom, his Life ... London 1933.

Loeffler, Wilhelm, Die Zürcher Medizinische Klinik 1833-1951. Zürich 1951.

Merked, Friedrich, Jacob Henle. Braunschweig 1891.

Mondor, Henri, Anatomistes et chirurgiens. Paris 1949.

Quincke, Heinrich I.: Edgar Goldschmid, Heinrich Quincke. Schweiz. Medizinische

Wochenschrift 44. (1945).

Schönlein, Jo. Lukas: Virchow, Gedächtnisrede. Virchow's Archiv, 1865.

Sigerist, H.E., Große Ärzte. 2. Auflage, München 1933.

Véron, Louis-DésIré: M.-E. Binet, Un médecin pas ordinaire: Le Dr Véron. Paris 1945.

Virchow, Rudolph: Briefe an seine Eltern. 2. Auflage, Leipzig 1907.

- Hirsch: Allg. Biogr. Lexikon ... Scheuthauer.

Weber, Fred. Parkes, Some Aspects of Death and Correlated Aspects of Life in Art, Epigram and Poetry, $4^{\text {th }}$ edition, London 1922.

\title{
Rennward Cysat als Naturforscher, Apotheker und Arzt (1545-1614)
}

Von P.Jung, Meggen/Luzern

Der Historiker und Staatsmann Rennward Cysat dürfte allgemein bekannt sein. Hier mögen die wichtigsten Daten dieses selten vielgestaltigen Lebens genügen.

Geboren 1545, als Sproß alten Mailänder Adels, der de Cesati oder Cisati. Sein Vater war schon in seiner Jugend nach Luzern ausgewandert, heiratete die Tochter des Ritters Rennward GölduIn, dessen Vorfahren aus Zürich kamen, stirbt nach fünf Jahren und hinterläßt eine Witwe mit drei Kindern, darunter RENNWARD. Ausreichende Mittel bestanden keine, die zwei Brüder starben bald, so daß Rennward allein übrig blieb. Anderthalb Jahre nach Vaters Tod geht die Mutter eine zweite Ehe ein mit Conrad von Lauffen. Auch er stirbt bald unbemittelt und hinterläßt seiner Witwe drei Kinder, so daß diese nunmehr die schwere Familienlast mit vier Kindern zu tragen hat.

RENNwARD entwickelt sich äußerst günstig, fällt durch Begabung und Fleiß auf, lernt als Autodidakt fremde Sprachen, besonders Französisch und Italienisch. Mit vierzehn Jahren, nach sechseinhalb Jahren Schulbesuch, kommt er zu den Apothekern Claduser und Hegner in die Lehre. Er beschäftigt sich viel mit Naturgeschichte, notiert sich bewährte Arzneivorschriften, geht vorhandener ein- 
schlägiger Literatur nach und wird so, der zeitgegebenen Entwicklung gemäß, Apotheker und Arzt.

1568 heiratet er Elisabetha Bosshart, des Ratsherrn JakoB Bosshart aus Freiburg im Üechtland, der schon seit 1547 Bürger von Luzern war. Die Ehefrau entwickelt sich als treffliche Gattin, schenkt ihm vierzehn Kinder und erleichtert ihm seine vielseitige berufliche und amtliche Inanspruchnahme. Cysat wird nämlich 1570 «Unterschreiber», nachdem er elf Jahre ausschließlich seinem Apothekerberuf gelebt. Er beginnt mit Sammeln und Ordnen der Staatspapiere; Tinte und Siegelwachs verfertigt er selber. Seine politische Karriere entwickelt sich rasch. Er wird Großrat, Comes palatinus, Eques aureatus, apostolischer Protonotar, empfängt den Kardinal Carlo Borromeo, mit dem er in lebhaftem schriftlichem Verkehr steht. So entwickelt sich C. mit größtem Erfolg zum Staatsmann von europäischem Ruf. Daneben funktioniert er gleichzeitig als «städtischer Schauspieldirektor», der die von Staats wegen aufgeführten, berühmten «Osterspiele» leitete.

Dies ungefähr der gedrängte äußere Umriß dieses vielgestaltigen Daseins. Uns interessieren hier seine naturhistorischen Forschungen und seine Tätigkeit als Heilkundiger.

Seine handschriftlich erhaltenen «Beobachtungen» überschreibt er mit «Observationes variae tam jucundae quam utiles de rebus physicis, naturalibus ac medicinalibus Renwardi Cysati. Amicis et posteris consultum». Diese Kunst (der scharfen Naturbeobachtung) habe er von Jugend auf gepflegt, schreibt er in dem Kapitel «De arte vel professione simpliciaria vel cognitione simpliciorum. «Sie ist mit mir ufgewachsen, allerzyt als sondere Lust und begierd gehapt.» Seine naturwissenschaftlichen Beobachtungen sind ungeheuer weitläufig und für seine Zeit jedenfalls recht genau. Er pflegt sie peinlich, sie begleiten ihn inmitten aller politischen Obliegenheiten und Kämpfe, denn «es ist nüt so gering oder kleinfügig in rerum natura, das nit auch verdiene, daß es in Achtung genommen werden möge». Deshalb fühlt er sich zu allem hingezogen, «was zu der Phisik und Medizin gehört» und widmet diesem Studium täglich eine halbe Stunde, die er sich von seiner Tagesarbeit abzwingt. Namentlich am Rigi, zwischen Vitznau und Weggis, ist er emsig tätig. In der Beschreibung De balneo lucelvia, das 1661 erschien, berichtet sein Enkel LEopold Cysat, wie der Großvater einen Katalog und ein Register «der Simplicien und Gewächsen» jener Gegend in mühevoller Arbeit angelegt. Deren Zahl schätzt er auf 800. Alle diese Aufzeichnungen sind handschriftlich in der Bürgerbibliothek in Luzern niedergelegt. Die einzelnen Namen der Pflanzen allein füllen mächtige Seiten, und es wäre wohl des Interesses eines Fachkundigen würdig, sich einmal ihrer anzunehmen.

Cysat schreibt ein besonderes Gartenbuch, das er mit eigenen Zeichnungen über Pflanzenanatomie ausstattet. Häufige Korrekturen und durchgestrichene Seiten sind Zeugen seiner gewissenhaften Beobachtung. 
In einer Anleitung Von der Multiplication etlicher Gewäxe gibt er Ratschläge für Pflanzen-, namentlich Beerenkultur und stellt in den Observationes de agricultura Vorschriften und Anleitungen über Acker- und Waldpflege auf. Weiter verbreitet er sich über Kristalle oder an anderer Stelle über Seide und Seidenwürmer. Ein weiteres Kapitel gilt der Geschichte, Kultur und erfolgreichen Pflanzung von Lauro-Cerasus. Er beschafft ihn sich aus Italien, angeblich den ersten in nördlichen Gegenden, der zur Blüte kam und sogar Früchte trug.

Die botanischen Beziehungen zu Italien finden ihren Ausdruck im Kapitel «Simplicia quae reperiuntur in monte Baldo ad urbem Veronam», eine Aufzählung von Pflanzen, die sich am Fuße des Monte Baldo, am Gardasee finden sollen, «über die Maßen große Kürbisse», offenbar Melonen.

Von seinen geplanten Pflanzungen im eigenen Garten spricht er im Kapitel «Simplicia adhuc acquiescenda pro horto meo in quantum fieri potest» und inwiefern seine Absichten von Erfolg begleitet waren, meldet er unter «Simplicia horti Mussegensis», einem speziellen Katalog seines eigenen botanischen Gartens bei seinem Hause auf der Musegg, wo er sogar Reben zu züchten und einen Weinberg anzulegen versuchte.

Der Gartenbau blieb ihm Studium, Belehrung und Erholung. 321 verschiedene Pflanzenarten pflegte er in seinem Garten, darunter manche in der Gegend noch wenig bekannte. Überall, wo er konnte, suchte er bei seinen zahlreichen auswärtigen Freunden um weitere Beiträge für seine Kulturen nach. Sein Sohn EmmanueL brachte ihm aus Padua die Wurzel der «Corona Imperialis». Aus Piemont soll C. die ersten Christian-Birnbäume nach der Schweiz verpflanzt haben, ebenso aus Frankreich die Äpfelsorte Carpentier. Blumen und Früchte wirkten verführerisch auf die benachbarte Jugend, so daß C. seine Maulbeer- und Pfirsichbäume mit «Klamern» schützen mußte. Er veredelte auch selbst gepflanzte Haselnußbäume und aus Plurs im Veltlin besorgte der Badearzt Columban eine Birnenart, die durch ihre mächtigen Früchte auffiel, so daß sie bei deren Reife durch «Stangen» gestützt werden mußte. In seinem Blumentrog vor dem Hause zogen schon früh im März die herrlichsten Blumen zahlreiche Beschauer an.

Seine landwirtschaftlichen Erfahrungen dienten allerdings nicht allein der wissenschaftlichen Beobachtung, denn neben seiner Gartenkultur trieb er auch Viehhandel. Überhaupt beschäftigte er sich viel mit dem Tierreich, schreibt von «Scheermüsen (Erdmüsen) und deren Bekämpfung.» Von «Raupen und Gewürmer», von Insekten, Bären, «Pfärden», vom Zähmen der wilden Tiere, von «Razen und Müsen» ist die Rede. In einem Kapitel «de animalibus infectis» läßt er sich über «Hummel und Hurnussen» aus. Ans Phantastische grenzen seine Äußerungen über Hühnerzucht. Auch den Störchen, die er in Luzern beobachtet, galt seine Aufmerksamkeit und im eigenen Garten hielt er Schildkröten.

Meteorologische Beobachtungen notiert er fleißig. Daneben beschäftigt ihn das Echo und der Ton der Glocken, aber gleich daneben folgt eine Nutzanwendung 
von «zerbrochenen Gläsern und ihrer Wiederherstellung». Eine ausführliche Beschreibung widmet er dem «Drachenstein», und stellt Betrachtungen an in einem Kapitel «vom Tonder», worin ihn die Gewittererscheinungen beschäftigen.

In der Nacht vom 18./19. September 1601 wurde ein gewaltiges Erdbeben am Vierwaldstätter- und Zugersee, wie übrigens auch in vielen andern Ländern, beobachtet. C. beschreibt es unter dem Titel «Von dem großen und erschrecklichen Erdbidem», wie es über die «ganze Eidgenossenschaft, Deutschland, Niederlande, Burgund, Italien bis gegen Piacenza und Parma» wütete. Geringe Wiederholungen, bis deren vier, sollen sich im Spätjahr, zuletzt im November 1601, ereignet haben.

Seine Lehrzeit bei den Apothekern Cuauser und Hegner in Luzern setzte er nach fünf Jahren bei einem Jugendfreund seines Vaters, Francesco Beccaria in Mailand, fort, wohin er 1564 übersiedelt, um ein Jahr lang zu bleiben. Das Lehrgeld wurde ihm wegen ausgezeichneter Eignung erlassen. Von Mailand aus besorgte er auch den Ankauf von Medikamenten für die Urner Ärztin DoRotheA von Mentlein.

Neben der vermehrten Gelegenheit zu Studium und Sammeln ihm noch unbekannter Pflanzen verschafft er sich neue Arzneimittel, stellt eigene Untersuchungen darüber an und prüft deren Heilwirkung, eine Tätigkeit, die er nach seiner Rückkehr in die heimische Apotheke mit vermehrtem Eifer fortsetzt. Eine Menge Rezepte in italienischer Sprache zeugen davon. Stets begleitet er seine Vorschriften mit dem Rat zu vorsichtiger Prüfung der Heilmittel und ihrem Gebrauch, damit kein Schaden entstehe. Die Produkte seines mit Liebe und Sorgfalt bei seinem Hause auf der Musegg gepflegten botanischen Gartens bieten ihm reichlich Material und Anregung.

Manche seiner Mitteilungen sind nicht frei von Aberglauben, so sehr er sich auch sonst dagegen wehrt und die Kurpfuscher mit Gift und Satire bloßstellt, sie auch wiederholt «Kalberartzet, Zauberer» schilt. Aber als Kind seiner Zeit bleibt C. vielfach derem Fühlen und Denken verhaftet und bewegt sich damit gezwungenermaßen oft in den krassesten Widersprüchen.

Davon ein paar Beispiele. Ein leerer Essigkrug, zu Weihnachten mit Wasser gefüllt, soll drei Monate später wieder richtigen Essig enthalten. Aber bei dieser Angabe spricht der Schalk aus C., denn er schließt sein Rezept mit der Bemerkung: «Darüber lasse ich nun die Experiens urteilen.»

Auf der Rückreise von einer Pilatustour am 31. Juli 1572 sticht ihn die Neugier, die allgemeinverbreitete Sage nachzuprüfen, wonach ein Gewitter entstehe, wenn man dem im See hausenden Pilatus durch Steinwürfe ins Wasser reize. Da aber kein Unwetter ausbrach, benützte C. diese Erfahrung, um über derlei Aberglauben Spott und Verachtung auszugieBen.

Anderseits kommt er vom Glauben an Drachen und an die Heilkraft vom sogenannten Drachenstein nicht ganz los. Dieser Wunderstein bildete lange Zeit eine allgemeine Sehenswürdigkeit, war meist im Besitz angesehener Luzerner Familien und wurde später im dor- 
tigen naturhistorischen Museum aufbewahrt. Es soll sich nach geologischer Feststellung um einen kugelförmigen, glatten Quarzit handeln. Auch die Existenz von «Erdmännchen» nimmt C. an.

An Gespenster glaubt er, läßt ein solches aus dem eigenen Haus durch den Pfarrer von Meggen für $1 \mathrm{Gl} .23 \mathrm{Sch}$. vertreiben. Auch den «höllischen Jäger mit den dreibeinigen Hunden» kennt er und beschreibt ein Gespenst, das in einer Sommernacht 1607 über Luzern wegging «mit grünlichem Schwanz, so lang wie der Platz». Auch im Januar 1608 will C. wieder ein solches beobachtet haben, das in gleicher Richtung wie das vorgenannte seinen Weg von der Egg über den Kornmarkt nahm. Sechs Tage vor dem Tode seiner Frau, die am 19. März 1614 starb, hört er, wie ein «Rumpeln» im oberen Korridor seines Hauses einen bevorstehenden Todesfall kündete. Ähnliches berichtet er vor dem Tod seiner Schwägerin, die 1594, starb.

Wie sehr er übrigens unter dem Eindruck des Mystischen stand, erhellt 1607 aus einem Ankauf von Leonardus Fioravantinuns De Novis Miraculis.

Eifrig dem Studium von Paracelsus, Philip Ulstadius und Bonifacius Pedemontanus ergeben und unter dem Einfluß des ihm befreundeten Schaffhauser Bürgermeisters Meyer, gab er sich auch mit Alchemie ab. Seine Aufzeichnung alchemistischer Beschriftungen und Auszüge, namentlich Rezepte aus den Schriften Theophrasts, geben davon Kenntnis.

Cysat, den begreiflicherweise schon die Sorge für seine große Familie stets plagte, unterlag den Versuchungen seiner Zeit, Gold herzustellen. «In 10 Tagen auf $100 \mathrm{~K}$. deren $25 \mathrm{zu}$ gewinnen» hatte er gehört, war aber bald von seinem Irrtum bekehrt. Er gibt ihn offen zu und warnt die Jugend ernstlich vor der Gefahr solchen aussichtslosen Unterfangens, das zur verheerenden Leidenschaft Anlaß biete.

Daß dem durch Staatsgeschäfte ohnehin Überlasteten der Wunsch, für seine Apotheke einen Nachfolger zu finden naheliegen mußte, ist ohne weiteres verständlich. So schickt er 1596 seinen Sohn Emmanuel nach Padua, um Botanik und Medizin zu studieren. Der botanische Garten dort bot besondere Anziehungskraft. Daneben aber wurde ihm der Spitalbesuch nahegelegt, um gleichzeitig medizinische Studien zu pflegen. Als aber zwei Jahre später (1598) EmmanueL zurückkehrte, mit bestandenem Examen und einer Apothekerlehre, nach des Vaters Überzeugung tüchtig ausgebildet, wurden diesem öffentlich verletzende Vorwürfe gemacht. Der Sohn sei noch zu jung für den Beruf des Apothekers, verfüge über eine mangelhafte Ausbildung, verdanke seine Ernennung zum Apotheker lediglich der väterlichen Protektion usw. Der Vater fühlte sich durch diese Vorwürfe schwer gekränkt, tröstete sich aber in einem Verse mit dem Schlusse:

«Kein Gwild schadt nit soviel Ihm wald, als tuth eine falsche Zunge kallt»,

Nichtdestoweniger lösen die schweren Aufregungen im Verein mit ständig sich mehrender Arbeit bei C. eine ernste Erkrankung aus, die ihn anfangs 1599 für zwei Monate darniederhielt. 
Das führt uns zur Betrachtung von R. Cysat als Arzt. Daß er nicht nur fleißig Medizin studiert hatte, sondern den Arztberuf auch ausübte, ergibt sich aus seinem bereits erwähnten minutiösen Rechnungsbuch. Dort trägt er unter anderm ein, daß ihm am 12. Juni 1603 die ärztliche Behandlung des «Dechan H. JoHANN Müller, später Chorherr zu Münster, vom Pabst Clemens dem VIII. eine goldene Medaille im Wert von 21 Fl. 3 Sch.» übermacht wurde. Ein anderer Posten in seinem Rechnungsbuch nennt unter dem 17. Januar 1594 «für Rat von Arzneikunst eine Einnahme von 7 Gulden».

Als 1583 eine Pilgergesellschaft von 33 Personen sich nach Jerusalem aufmachte, übergab ihnen C. ein kleines Buch mit Gesundheitsregeln, namentlich mit Rücksicht auf die drohende Pest. Tatsächlich kehrten denn auch alle nach zwei Jahren wohlbehalten zurück mit einer einzigen Ausnahme, indem ein Pilger den Tod auf der Insel Cypern fand.

Eifrig pflegte C. mit den bedeutendsten Ärzten seiner Zeit, mit Felix Platter in Basel, von Muralt in Zürich, Ludwig Kiel (Carinus) in Basel, kollegialen Verkehr, von letzterem notiert C. ein Rezept gegen Epilepsie, während Platter sich 1595 für ein überlassenes «Traktätlein» bedankt.

Cysats ärztliche Schriften sind außerordentlich vielseitig. In einem Artikel, «De Sympathia» behandelt er ihren Einfluß auf Mensch und Tier. In ähnlicher Richtung bewegt sich ein mit zahlreichen Beispielen belegtes Kapitel «von der Imagination». Auch der Aufsatz «von den Besessenen» und ein solcher "pro morbo Melancholia» mit beigefügten Rezepten gehören hieher. Es wäre aber ein Irrtum anzunehmen, daß er bei der Wiedergabe von Mitteilungen und eigenen Beobachtungen irgendwelche Systematik verfolgte. C. schrieb offenbar alles nieder, wovon er las oder hörte. So spricht er über «seltenes hohes Alter», ferner von der «Degeneration des Menschen», stellt Betrachtungen an über Blinde und sterbende Menschen. In den «Komplexionen des Menschen» berührt er die vier Temperamente und vertritt in einem Kapitel «von unglicher Wirkung der Natur» eugenische Ideen.

In den Consilia Medicinalia in Curandis Morbis stellt er eine Reihe von Rezepten und Ratschlägen zusammen. Wir finden da «eine Aqua contra Epilepsiam», ein «preservat ab apoplexia». Die verschiedenen Balsame und ihre Herstellung beschäftigten ihn ebensosehr wie die «Aqua mirabilis» und dessen Zusammensetzung aus elf Medikamenten. Gegen «dolorem stomachi» oder «contra podagram» schlägt er Mittel vor, erteilt Ratschläge bei «erfrornen Gliedern», wie er sich bemüht, den Geburtschmerz zu erleichtern. Seiner Gattin verschreibt er in einem Artikel «Consilium pro uxore mea pregnante: pulv. Cinammom». Unter den angegebenen Vorschlägen und Rezepten fallen Mitteilungen von Dr. Byger in Zürich auf.

Gegen die Scharlatane wendet er sich mit zunehmender Vehemenz. In einem Hexameter überschüttet er sie mit Hohn und Spott. 
Ein Kapitel «Doctores selbs krank» behandelt ironisch die leidenden Ärzte, die zwar ihre Patienten zu heilen imstande sind, ohne sich selbst von ihren Leiden befreien zu können. Eigene üble Erfahrung mag ihn dabei geleitet haben, denn die «Sciatica genuum, die mich etliche Mahle streng geplagen», mag ihn zu diesen Betrachtungen verleitet haben.

Für das Bad Fideris, obwohl «er nie dort gewesen», sammelt er Erfahrungen von Patienten, die dort Heilung gesucht, Ratschläge zu zweckmäßigem Kuraufenthalt.

In Anlehnung an eine Mitteilung Konrad Gessners schreibt Cysat «Von Fruchtbarkeit der Frauen dieses Landes» und berichtet als Sonderlichkeit über eine Frau, die mit 49 Jahren noch ein Kind zur Welt brachte. Daneben erwähnt er eine Geburt von Vierlingen in Kriens, die angeblich alle lebend und gesund waren.

In seinen Manuskripten findet sich auch ein «Consilium contra Ophtalmiam» von Felix Platter. Hager gibt ihm Ratschläge und Rezepte zur Behandlung von Ischias und Melancholie, und von Dr. Christoph Byger in Zürich erhält C. sieben Rezepte nebst Diätvorschriften bei Kopfschmerzen, deren Symptomatologie besprochen wird.

Wie wirr und bunt Cysats ärztliche Bemühungen sich oft gestalteten, mag seine Mitteilung zeigen, wie er in Italien anscheinend Wein herstellen gelernt und nun von einem Mittel berichtet, um «Weintrinken zu lehren» und anderseits durch «Capreoles vitis» Trunksucht zu heilen.

So gleitet C. in das Gebiet des Phantastischen und Abergläubischen, wie wir das aus seiner Beschreibung von der Wirkung des «Adlersteins» gegen alle denkbaren Beschwerden hören. Dieser Stein wurde am Hals getragen. Mag C. vielleicht dabei einer Konzession an die damalige Volksmedizin gefolgt sein, so finden wir die Idee auch heute noch oft genug vertreten, begegnen wir doch immer wieder Leuten, die aus prophylaktischen Gründen ständig Zwiebeln mit sich herumtragen oder gar Kartoffeln versteckterweise in ihre Kleider einnähen, um sich damit vor Krebs und Rheuma zu bewahren.

«Von einer wunderlichen Blutstillung», die einem spanischen Bischof gelang, macht C. Mitteilung, ebenso von der Heilung eines Hundebisses, wie ihm überhaupt das Leben und Treiben der Hunde stark beschäftigte. Er hat ihnen ein besonderes Kapitel «De Canibus» gewidmet.

C. war von einem unermüdlichen Sammeleifer beherrscht, der ihm Anlaß gab, alle auch in der damaligen Zeit kaum glaubhaften Mitteilungen festzuhalten. So schreibt er beispielsweise über «ein Kind, von einem Mann geboren». Offenbar trug C. alles an interessantem zusammen, dessen er irgendwie habhaft werden konnte. So allein läßt sich diebunte Mannigfaltigkeit seiner Zusammenstellungen erklären. Das «Schnarchen» beschäftigt ihn ebenso wie Beobachtungen an Trunkenen, denen Betrachtungen über die «seltsame Natur des Menschen», psychologische Beobachtungen über dessen Eigenarten, so über die «Hartnäkigkeit» folgen. Eingestreut sind Mitteilungen über «große Menschen», womit er offenbar Fälle von Riesenwuchs meint und wobei er eigene Beobachtungen mit fremden Mitteilungen vermengt. 
Die eigene Erkrankung zwang C. erstmals 1580 zu einer Badekur, die er an einem heute vergessenen Badeort Rothen bei Emmenbrücke absolvierte. Wie wir des öftern hören, litt C. damals vor allem an «Rheuma», klagte aber auch über Magen- und Darmbeschwerden, ferner plagte ihn ein Augenleiden, das unter anderm auch von Felix Platter behandelt wurde. Auch Giger, Hager und Quentzi hatten sich vielfach um Cysats Klagen bemüht. Das Bad in Rothen soll Alaun und Salpeter enthalten haben und wurde hauptsächlich gegen Hautund Gelenkskrankheiten verwendet. Dieser ersten Badekur verdanken wir Cysats Badevorschriften. Solche sogenannte Badebücher existierten damals verschiedene. Durch deren Studium bereitete sich C. auf seine Kur peinlich genau vor, und was er in früheren Vorschriften durch eigene Erfahrungen bestätigen kann oder ablehnen zu müssen glaubt, das veranlaßte ihn zur Aufstellung seiner Anleitungen.

C. blieb anfänglich 2 Stunden täglich im Bade und stieg dann jeden folgenden Tag um eine Stunde bis zum Maximum von 7 bis 8 Stunden, dann absteigend, bis abschließend mit 2 Stunden Badedauer. So kam er auf eine Badeanwendung von total 152 Stunden, eine Konzession an die auch heute noch verbreitete Auffassung: Je länger das Bad, um so nachhaltiger der Erfolg. Leib und Seele schenkte er dabei die größte Aufmerksamkeit, und vom zweckmäßigen Verhalten leitet er den guten Erfolg der Badekur ab.

Am ersten Tag läßt er nur «ein halb Quertlin» trinken und jeden folgenden Tag etwas mehr, durch acht Tage hindurch, dann decrescendo, bis man ganz auf hört. Das Wasser darf nicht kalt sein, sondern es empfiehlt sich die Überwärmung und man soll es «langsam und sittig hinabsupflen». Da man damals in den Bädern vieldach ein Schlemmerleben führte, sieht er sich zur Mahnung gewisser Vorsicht verpflichtet. Allerdings will er nicht, daß der Kurgast Hunger oder Durst leide, aber er soll sich vernünftige Zurückhaltung auferlegen. Er rät zu «mittelmäßigem und lauterm» Wein, zum Nachtessen soll er «dünnrot» sein. Diese Vorschrift mag Bezug haben auf den damaligen Gebrauch, den Wein zu kochen und mit allerlei Zutaten zu versehen, eine Art Glühwein. Von Brot wünscht er, daß es einen Tag alt sei, dann folgt in der Speisenreihe: die Suppe und leicht verdauliches Fleisch, von «jungen Zicklen», Kälbern und Lämmern, auch Geflügel ist erlaubt. Verboten dagegen: Wasservögel; Wildbret ist nur von jungen Hirschen und Rehen zulässig. Zutaten, wie Zwiebeln, Knoblauch, Rettich, Senf, Hasenfleisch und solches von alten Rindern, Milch und Salat, sind verpönt. Wohl aber werden empfohlen: Rosinen, Zibellen und große Weinbeeren, weichgesottene Eier, Hechte, Egli, Karpfen, Forellen, Erbsen, Gerste und Haberkörner, Petersilie, Fenchel. Lattich bleibt für jene mit einem «guten und nicht zu kalten Magen» reserviert. Der Salat, soweit er zulässig, wird mit frischer Butter oder Baumöl angemacht. Empfohlen sind Kapern, Zitronen, Orangen und Lemonen. Und beschlossen soll das Mahl werden mit «Diaci toniton, Kondit oder Marzapan». Alle sogenannten tierischen Innereien und gesalzenes Schweinefleisch sind den Kurgästen verboten, ebenso wie der Käse. Sofern er solchen zuläßt, muß er möglichst geruchlos und nicht gesalzen sein. Frische Früchte, wie z. B. Kirschen, verbietet er. Namentlich auch, weil sie meistens unreif auf den Markt kommen. Reife Erdbeeren mit Zucker und Wein sind erlaubt, ebenso die «Wiexeln», also sogenannte Weichselkirschen. Vor neuem Obst wird gewarnt, ausnahmsweise sind Zwetsch- 
gen zulässig, Äpfel und Birnen aber nur gekocht oder gebraten. Für die Heimreise soll man schönes Wetter wählen, da man sich sonst leicht erkältet.

Am 23. Juni 1580 kehrte C. mit seinem Badefreunde Nikolaus Cloos aus dem Bad Rothen nach Hause zurück. Neben diesen Badevorschriften ist es das Pestbüchlein, das uns Cysats Bemühungen und Arbeit als Arzt am nächsten bringt.

Von diesem Pestbiichlein (1594) existiert die Handschrift und aus dem gleichen Jahr ein Druck durch Abraham Gemperlin zu Fryburg im Üchtland. Eine neue Auflage erschien 1611 bei Witwe AnNa Jergin in München. Eine Pestverordnung bestand übrigens bereits seit 1580, sie wurde von CYSAT erneuert und ergänzt. Durch behördlichen Erlaß wurde C. 1596 mit der Durchführung der Vorschriften betraut. Auch von Quentzi, Hager und F.Platter erhielt C. Ratschläge zur Pestbekämpfung.

Seine Verordnung betitelt sich Reglement der Gesundheit und Ordnung in Pestilenzischen Zytten. Es gliedert sich in mehrere Abschnitte. In der «Vorbereitung» gibt er allgemeine Ratschläge, z. B. warnt er vor übertriebener Angst, rät zur Körperpflege und zur Förderung der Darmentleerung. Der folgende Abschnitt bespricht die natürlichen Mittel; im wesentlichen sind es Diätvorschriften: Warnung vor schwerem Fleisch, Kutteln, Blut- und Leberwurst, scharfem Käse. Mäßigung in Speis und Trank, ausreichender Schlaf, Vermeidung von Aufregung werden dringend empfohlen. Die Prophylaktika gruppiert er verschieden für Arme und Vermögliche, nennt Kräuterabsude, Tees usw.

Der dritte Abschnitt betitelt sich: «Wie man sich im Angriff dieser Krankheit halten soll.» Wichtig ist die Förderung des Schweißausbruchs, namentlich auch bei Schwangern, die Sorge für Flüssigkeitsersatz, die Warnung vor Most, kalter saurer Milch und übertriebener Wärme. Großen Wert legt er auf peinliche Sauberkeit der eigenen Person und des Gesindes.

Der Eiter aus den Pestblasen soll in fließendem Wasser oder in der Erde unschädlich gemacht werden. Unter Hinweis auf die großen Gefahren der Krankheit mit drohendem Tode werden soweit möglich Vorschläge zur Isolierung der Kranken gegeben. Deren Abfälle und Exkremente müssen beseitigt werden, ebenso tote Haustiere, Mäuse und Katzen. Ferner werden Räucherungen mit Weihrauch, Rosmarin und Lavendel empfohlen.

Als Heilmittel nennt C. für die Armen: «Loubstickel oder Strenzenwürtzen, Reckholder, Eicheln, dürre Apfelrinden oder Schnitz.» Den Begüterten wird Rosenwasser mit weißem Wein zum Riechen und Benetzen von Taschentüchern und Handschuhen angeraten. Schwangere sollen sich der Muskatnuß bedienen. Für die Männer allein kommt zweimal wöchentlich «ein halb Quentlin venedrischer Tryax [Therak] oder Mithridats» in Betracht. Die Armen sollen Reckholderbeeren und Baumnußkernen in Essig, morgens nüchtern, nebst Zieger und Knoblauch einnehmen. 
Endlich folgt der Vorschlag eines Amuletts unter Berufung auf bewährte Ärzte und Doktoren. Die Herstellung erfolgt nach dem angegebenen Rezept: «Ausgehöhlte Haselnüsse werden mit Quecksilber gefüllt und mit spanischem Wachs verschlossen.» Das Mittel wird auf dem Körper getragen und sei vorrätig in den Apotheken.

Nach Ablauf der Erkrankung hat eine Hausräucherung mit Schwefel zu erfolgen. Ist der Kranke außer Gefahr, frühestens nach sieben Tagen, darf er etwas Wein nehmen, «aber bescheidentlich». Schließlich ist beigefügt eine «Aderlaßordnung an Orten und Enden, nachdem sich dann uff den schon Verzichten der Gebrest erzeigt oder uffwürfft».

U̇ber allem aber steht die Warnung vor «Schärern und Badern», dringend wird der Beizug eines Arztes angeraten.

Wegen schwerer rheumatischer Krisen ist C. gezwungen, im Jahre 1594 wiederum, vierzehn Jahre nach seiner Kur in Rothen, zum Kurgebrauch aufzubrechen. Er wählte diesmal Baden im Aargau. Diese Kur fällt in das gleiche Jahr, wie die Herausgabe des Pestbüchleins. Auch diesmal scheint C. für seine Beschwerden von Erfolg begünstigt. Wenn sie ihn auch nie ganz verlassen und er oft über sie klagt, so kann er doch während sieben Jahren einer weiteren Kur sich entschlagen. Dann aber setzen schwere «rheumatische» Attacken ein. Diesmal sucht er 1601 den Kurort Lützelau auf, damals ein Bad zwischen Weggis und Vitznau. Wie sein Enkel in der Beschreibung des Bades bemerkt, ist es «so genannt ihr kleine wegen» und es wird empfohlen mit dem Verse: «Schmerzhafte Prästen und Zuständ, vertreibt dies edle Bad behänd.» Auch C. der Großvater hat dem Badeort eine ausführliche Beschreibung gewidmet mit Anleitung zu zweckmäßigem Gebrauch und über die zu erwartende Wirkung. Nach dem Bericht des Enkels hat C. dort eine gute Kur gemacht und nachher wesentliche Besserung von seinen Beschwerden verspürt. Daß er übrigens dort nicht ausschließlich seinen früher aufgestellten Kurvorschriften gemäß lebte, geht aus dem Bericht des Enkels hervor, der von ständigen botanischen Streifzügen des Großvaters berichtet und auch ein Lobgedicht erwähnt, das dieser auf den Kurort verfaßte. Sein leidender Zustand macht aber offenbar weitere Fortschritte, und eine Reise, die er 1606 nach Bayern machte, ist ihm ganz schlecht bekommen. C. wurde «brustkrank» und stand deshalb in Behandlung von Dr. Burckhart. Er tritt 1613 am 19. Juni, offenbar schon schwer krank, während 25 Tagen eine weitere Kur in Lützelau an. Sie bringt ihm eine gewisse Linderung, offenbar ohne den Verlauf seines Leidens nachhaltig zu beeinflussen. Wenn er auch dank seiner Energie seine politische Betätigung wieder aufnahm, so stellte sich doch zunehmender Zerfall ein. Wie bereits erwähnt, glaubte er, das «Künden» eines bevorstehenden Todesfalles in der Familie zu hören. Am 19. März 1614 starb seine Frau, für C. der schwerste Verlust. Offenbar ahnte er nicht, daß er sie nur um weniges überleben würde, denn noch vom 20. April 1614 datiert eine Eintragung unter seinen Einnahmen und 
schon fünf Tage später folgte C. seiner Gattin im Tode. Seine Grabstätte hatte er längst im Chorherrenstift Hof erworben. Die Regierung ehrte sein Andenken durch das Geschenk eines «hundertlötigen Silbergeschirrs» an seine Hinterbliebenen. A. von Haller hat ihn als den großen Cysat gefeiert.

\section{Thomas Harriot (1560-1621) fumeur de pipe, victime du cancer?

\author{
Par Juraj Körbler, Zagreb
}

Déjà en 1739 Johann Jacob Holland supposait dans sa dissertation Dissertatio inaugur. med. chir. sistens Carcinoma labii inferioris absque sectione persanatum, qu'il y a une relation causale entre l'usage de fumer la pipe et l'apparition du cancer de la lèvre inférieure. En 1795 Samuel Thomas Soemmering (1755-1830) précise que ce n'est pas la fumé qui provoque la cancerisation mais les sucs qui se forment dans la pipe. R. Melzer ${ }^{1}$ croit avoir observé, qu'une telle cancerisation n'arrive jamais dans l'Orient ou on fume le tabac cru sans aucune préparation préalable. Une observation qui est d'ailleurs tout autre que juste. Il est intéressant de noter à ce sujet, que la première observation d'un cancer de la lèvre inférieure comme suite de l'usage de fumer la pipe est bien plus ancienne. Elle date de la même époque comme l'usage même de fumer la pipe en Europe, cèpendant la relation existante entre la pipe et le cancer n'était pas encore reconnue. Il s'agit du cancer de la lèvre inférieure dont était atteint Thomas Harriot, savant naturaliste anglais. BERnard JAFFe donnait récemment un éloge de Harriot dans son livre sur les savants americains: Men of science in America ${ }^{2}$.

L'intérêt des Anglais pour les pays d'outre-mer apparaissait relativement tard. Les Espagnols possédaient déjà à cette époque des empires immenses sur le nouveau continent. Après Humphry Gilbert, qui périt en mer sans avoir réussi de fonder une colonie en Amérique du Nord, son demi-frère Sir Walter Raleigh obtenait le 25 mars 1584 de la reine Elisabeth un patent et une licence pour la colonisation dans le Nouveau Monde. C'est pourquoi Raleigh est consideré comme un des "founders of America".

Raleigh organisa une expédition sous le commandement de Amados et BARLow pour explorer les régions entre Florida et New Foundland. Avec des rapports enthousiastes sur les richesses de ces pays, cette expédition à son retour en Europe amenait deux Indiens, Manteo et WANchese, pour s'en servir des interprètes dans les expéditions qui devront suivre. Pour mieux explorer tous les richesses de

${ }^{1}$ Jenaische Annalen 2, 480 (1850).

2 New York (1944). 